\title{
GLOMERULAR DISEASES \\ Regulation of IgA production by TBK1 prevents nephropathy
}

Immunoglobulin class switching to the $\operatorname{IgA}$ isotype is negatively regulated by the serine/threonine-protein kinase TBK1, according to new data from a study published in Nature Immunology. IgA has an important role in mucosal immunity and its dysregulation has severe pathological effects, including IgA nephropathy and kidney dysfunction.

The researchers used Tbk1-BKO mice, in which $T b k 1$ is deleted specifically in $B$ cells, to investigate the function of the kinase in vivo. "Unlike conventional Tbk1 knockout mice, which are embryonic lethal, Tbk1-BKO mice have no survival problems" explains study author Shao-Cong Sun. B-cell-specific Tbk1 knockout did not affect B-cell development or maturation. However, following immunization, Tbk1-BKO mice produced significantly more antigen-specific IgA than did wild-type controls. Nonimmunized Tbk1-BKO mice aged $>12$ weeks had significantly higher concentrations of serum IgA than age-matched wild-type controls. After 8 months of age, Tbk1-BKO mice showed increased levels of autoantibodies to antinuclear antigen and double-stranded DNA as well as signs of nephropathy, including antibody deposition in glomeruli and increased concentrations of urinary protein and serum nitrogen and creatinine. These findings suggested a critical role of TBK1 in the regulation of $\operatorname{IgA}$ production and the prevention of nephropathy.

In vitro, TBK1 deficiency promoted the induction of $\operatorname{IgA}^{+} \mathrm{B}$ cells in response to concurrent stimulation with transforming growth factor (TGF)- $\beta$ and costimulatory molecules, indicating a role of the kinase in the control of IgA class switching. Further experiments showed that TKB1 negatively regulated class switching to IgA by attenuating the noncanonical

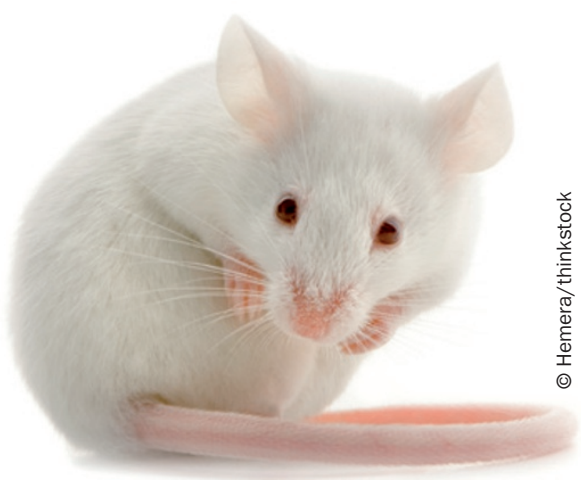

activation of nuclear factor $\kappa \mathrm{B}(\mathrm{NF} \kappa \mathrm{B})$ signalling, which has an important role in B-cell survival and antibody production. In B cells, activated TBK1 phosphorylated NIK-a kinase with a critical role in the induction of noncanonical NFKB signalling — resulting in its degradation.

"Our work identifies TBK1 as a crucial regulator of IgA production and also demonstrates a role for TBK1 in controlling the activation of noncanonical NFkB signalling" concludes Sun. "Since TBK1 is currently known as a kinase that mediates type I interferon induction in innate immunity, our findings are unexpected and substantially advance our knowledge regarding TBK1 function and IgA production."

In the future the researchers plan to investigate whether TBK1 has a role in the regulation of $\operatorname{IgA}$ induction in response to antigen, and if so, whether the activity of the kinase could be manipulated to improve the efficiency of mucosal vaccination. "This possibility is suggested, but not directly addressed by our current work" says Sun.

Ellen F. Carney

Original article Jin, J. et al. The kinase TBK1 controls IgA class switching by negatively regulating noncanonical NF-kB signaling. Nat. Immunol. doi:10.1038/ni.2423 